\title{
Acción vs Representación: el 15-M y su repercusión en la ciudad
}

\author{
Mayka GARCÍA-HÍPOLA y María BELTRÁN ROdRÍGUEZ \\ Departamento de Teoría y Proyectos en Arquitectura y Urbanismo \\ Escuela Politécnica Superior / Universidad CEU San Pablo \\ mghipola.eps@ceu.es y maria.beltranrodriguez@ceu.es
}

Recibido: 01/03/2013

Modificado: $22 / 04 / 2013$

Aceptado: 10/05/2013

\section{Resumen}

Este artículo analiza la relación entre el espacio público -lo físico- y la acción ciudadana -lo social-. Se utiliza como caso de estudio el Movimiento $15-M$, encuadrado dentro del movimiento global de ocupación del espacio público. Se examinan la auto-organización de los ciudadanos gracias al uso de nuevas tecnologías y su capacidad de acción y transformación del espacio público, así como el fenómeno de ocupación y de poder. ¿Cómo de público es verdaderamente el espacio público? La investigación se realiza desde las dos vertientes opuestas que intervienen en él: lo regulado y legal (sistema de planeamiento actual) y lo espontáneo y con frecuencia ilegal (movimientos sociales). Como conclusiones se plantean posibles vías de diseño para lograr un equilibrio entre el espacio público como lugar de representación y de acción, como espacio institucional e insurgente, a medio camino entre un diseño rígido o regulado $y$ uno flexible $y$ espontáneo.

Palabras clave: movimientos sociales, ocupación, espacio de acción, institucional, insurgente.

Title: Action vs Enaction: The 15-M and its Reverberation in the City Abstract

This article seeks to analyze the relationship between public space -the physical- and civic action -the social-. It uses the $15-M$ Movement as a case study, framed within the global occupation movement. It will examine the self-organization and the use of new technologies and their potential for citizens' action and transformation of public space, as well as the phenomenon of occupation and empowerment. How public is public space? The research focuses on the two opposing actors involved in public space: the legal and regulated (current planning system) and the spontaneous and often illegal (social movements). The conclusions lead towards ways to achieve a balance between the design of a public space as a place for representation and for action, as both an insurgent and institutional space, halfway between a rigid and regulated design and a flexible and spontaneous one.

Keywords: social movements, occupation, space for action, institutional, insurgent. 


\section{Índice}

1. Introducción

2. Puerta del Sol: antes / ahora / después

3. Los efectos del Movimiento 15-M: Acciones

3.1. La capacidad de auto-organización (Organizar)

3.2. El fenómeno de ocupación del espacio público (Ocupar)

3.3. El poder de transformación (Transformar)

4. Lo espontáneo se abre paso por lo institucional

4.1. El marco legal

4.2. Los movimientos sociales

5. Conclusiones

\section{Introducción}

Las marchas silenciosas de protesta por las calles de Leipzig en Alemania en 1989 fueron el comienzo hacia el final de la Guerra Fría. Las marchas estudiantiles todos los lunes por las calles de Belgrado en 1996 y 1997 fueron un condicionante importante para el restablecimiento de la democracia en Serbia. Las protestas pacíficas de madres contra la dictadura militar en Argentina, cada jueves de 1977 a 2007 en la Plaza de Mayo en Buenos Aires son otro ejemplo de cómo las concentraciones valientes y simbólicas en el espacio público han contribuido a un futuro mejor para los ciudadanos. La historia del mundo está llena de ejemplos similares que subrayan la importancia del espacio urbano como lugar de encuentro, a todos los niveles, desde las charlas a las demostraciones con gran repercusión. (Gehl 2010: 157) ${ }^{1}$

La concepción y el uso del espacio público puede variar según el lugar y la cultura, pero una constante a lo largo de la historia es que los espacios públicos son el lugar de nacimiento de las protestas democráticas, los movimientos, las reformas y las revoluciones como ocurrió con la vuelta a la democracia en Belgrado- como vemos en la cita anterior. El espacio público es un lugar donde la gente puede protestar pacíficamente de forma independiente o colectiva para cambiar las cosas. En ciudades de todo el mundo, la gente va a los espacios simbólicos de la ciudad para ser visto y oído. Prueba del poder de las manifestaciones en lugares públicos, son las represalias policiales a veces sufridas por sus participantes, su catalogación por parte de las fuerzas políticas en el poder como "amenaza de salubridad pública" y la imposición de nuevas normas y reglamentos para expulsar a los ocupantes de ese espacio ${ }^{2}$.

\footnotetext{
${ }^{1}$ Todas las traducciones han sido realizadas por las autoras del artículo.

2 Las actuales ordenanzas cívicas de Madrid castigan, entre otras actividades, la mendicidad (actividad con la que se asoció en múltiples ocasiones el asentamiento de Sol por parte de algunos políticos y medios informativos) o las acampadas en lugares públicos. Limitan así los derechos y libertades de algunos colectivos sociales. Es de destacar que el derecho de reunión está reconocido y regulado en la
} 
Los movimientos sociales de protesta surgen siempre en épocas de crisis, cuando los ciudadanos no están conformes o no se sienten representados en su país/ciudad por el sistema económico, político, ideológico o social. Actualmente es imposible hablar y actuar en la ciudad de hoy, si no se analiza su respuesta a las distintas crisis (económica, social, ecológica...). Durante décadas hemos crecido descontroladamente, sin tener en cuenta dos factores básicos. El primero, la red de interdependencia entre todos los componentes que hacen funcionar una ciudad. El segundo, los límites de crecimiento urbano. Las ciudades tienen unos límites (Herrero López 2012). Nos encontramos por lo tanto ante una situación general de estrés en términos de tiempo, energía y espacio ${ }^{3}$. Sólo si entendemos esta problemática general, podremos llevar a cabo una regeneración urbana -así como social, política y económica- profunda.

Una vez entendida esa situación global, hay que entender también el modelo de ciudad aplicado y las políticas llevadas a cabo en los últimos años en Madrid -contexto similar al de otras ciudades españolas-. Madrid se ha basado en un modelo liberal del "todo urbanizable ${ }^{\prime 4}$, que ha requerido de fuertes dosis de intervención pública sobre el territorio, no siempre de forma homogénea y socialmente equilibrada. Éste es el escenario en el que ha hecho su aparición la crisis y que sitúa a la ciudad de Madrid en una posición particularmente vulnerable, al haber basado su crecimiento en el sector inmobiliario y la construcción, y haberse financiado gracias a unos niveles de crédito insostenibles.

El movimiento $15-\mathrm{M}^{5}$ surge por lo tanto en dicho contexto urbano de desigualdad territorial y social, en mayo de 2011, mediante la ocupación de la Puerta del Sol, símbolo de la protesta contra los partidos políticos mayoritarios y contra las decisiones económicas y políticas adoptadas en España en los últimos tiempos. La protesta fue especialmente simbólica por la ocupación física -y espontánea- de un lugar emblemático ${ }^{6}$ de la ciudad que acostumbra a ser controlado por normas rígidas, a modo de plaza dura, fiel reflejo de la sede de la Presidencia de la Comunidad a la que sirve de

Constitución Española como derecho fundamental. Algunos artículos de ordenanzas frenan el derecho de reunión, de participación y manifestación.

3 Ver datos del Centro Complutense de Educación e Información Medioambiental (CCEIM).

${ }^{4}$ Producto de la Ley del Suelo de 1997.

${ }^{5}$ Movimiento ciudadano creado a partir de una serie de protestas y acampadas pacíficas en la Puerta del Sol en Madrid el 15 de mayo de 2011. Promueve una democracia más participativa y critica el excesivo poder de los dos principales partidos políticos españoles, de los bancos y las corporaciones. El Movimiento 15-M es conocido en las redes sociales mediante el hashtag \#spanishrevolution.

${ }^{6}$ Lugar dónde se encuentra el $\mathrm{km}$. 0 del país, o dónde se celebran las míticas campanadas de Fin de Año. 
antesala. No sólo se produjo una ocupación y consecuente transformación física del espacio (capacidad ciudadana para aportar, construir y articular servicios de primera necesidad como comedores populares), sino una reivindicación de lo público en la ciudad -espacio símbolo de regeneración social- y una auto-organización mediante sistemas más deliberativos y participativos, que aún hoy siguen vivos a través de las infraestructuras sociales creadas en múltiples barrios madrileños.

Esta crisis urbana y los graves riesgos de fractura social que conlleva pueden abrir posibilidades para un verdadero cambio de rumbo. Los conflictos, resistencias y malestares ${ }^{7}$ anuncian que la crisis es también una oportunidad de cambio, de reinvención del espacio público y sus usos, y una gran oportunidad para recuperar el "derecho a la ciudad" y para el desarrollo de una democracia urbana.

\section{Puerta del Sol: antes / ahora / después}

La Calle General [Global Street] necesita decisiones; necesita ocupación. Cuando hablo de calle general, me refiero no sólo a un espacio donde poder manifestarse, sino también donde aquéllos que no tienen acceso a los procesos formales de poder, puedan ejercer lo social y lo político. La calle urbana como espacio público ha de ser diferenciada de la plaza o avenida de tradición europea, un espacio para las prácticas rituales. (Sassen 2011: 569)

Según Sassen, los espacios públicos, la calle -que incluye también plazas y cualquier espacio urbano abierto- ya no hace referencia sólo a lo físico (de tradición europea milenaria) sino que es el lugar donde se recoge la voz ciudadana; siendo simultáneamente un foro físico (de herencia griega y romana) y uno virtual. El estudio de la ocupación de la puerta del Sol y del Movimiento 15-M se hace desde ese nuevo punto de vista ${ }^{8}$, haciendo una distinción entre los espacios que dan lugar a nuevas formas de lo social y de participación ciudadana (lugares de acción), frente a los espacios de carácter político, de representación - piazzas tradicionales ${ }^{9}$ - (lugares de representación).

\footnotetext{
7 Además del movimiento $15-\mathrm{M}$, ha habido otros movimientos de protesta social y salida a la calle de diversos colectivos: personal docente, sanitario, de justicia, minusválidos, etc.

8 Para este nuevo enfoque de estudio del espacio público cabe destacar los conceptos de "vita activa" (Arendt 1958), "activismo de la esfera pública" (Habermas 1997) o "transformaciones del espacio público en la sociedad contemporánea" (Innerarity 2006).

${ }^{9}$ Hace referencia al uso cotidiano de las plazas europeas desde el Renacimiento como lugares sociales de exposición y consumo, más que de acción social.
} 
La Puerta del Sol es un espacio público occidental, herencia del ágora griega ${ }^{10}$ y la res-pública romana ${ }^{11}$, símbolos de espacio de reunión y nacimiento de la democracia. Es un espacio que ha ido evolucionando desde el medievo ${ }^{12}$ (plaza asimétrica de uso comercial), pasando por el Renacimiento ${ }^{13}$ y los siglos siguientes cuando imperaba lo estético y formal frente a lo político y social ${ }^{14}$-, hasta llegar a la era contemporánea ${ }^{15}$.

En el caso de la Puerta del Sol en Madrid, tanto física (forma) como funcionalmente (acción), se observa este patrón evolutivo desde la Edad Media (figura 1): primero, espacio irregular que servía como mercado y entrada a la villa fortificada de Madrid en el siglo XVI (un punto en el mapa); luego, amplia calle que unía la ciudad nueva con la vieja en el siglo XVII (una línea en el mapa); más adelante, aparcamiento y nodo de tráfico durante el siglo XIX y parte del XX; y hoy, una plaza peatonal (plano en el mapa) que se utiliza para eventos y reuniones sociales.

${ }^{10}$ El Ágora se convirtió en el lugar clave para actividades políticas, económicas y sociales que giraban en torno a la democracia. Se creó un fuerte vínculo entre el ágora -escenario al aire libre para el debate- y la vida política que se llevaba a cabo en reuniones al aire libre. A medida que este sistema de gobierno fue desapareciendo, el significado de vida política fue quedando relegado a vida social (Arendt 1958).

11 Siguiendo la tradición griega, el foro romano constituye un espacio para actividades públicas y políticas, aunque se incorporan el mercado y el comercio (la unión de política y economía).

12 En las ciudades medievales los mercados se convirtieron en los protagonistas de las plazas, que variaban en forma y tamaño, según sus usos, dando lugar a dimensiones asimétricas y únicas.

${ }^{13}$ Plaza entendida como obra de arte, no como espacio funcional para necesidades sociales.

${ }^{14}$ Aunque sí acogieron las ejecuciones como actos públicos, como durante la Revolución Francesa.

${ }^{15}$ En las primeras décadas del siglo $X X$, los regímenes autoritarios utilizan la plaza como un contenedor, y se diseñan lugares de reunión masiva, centros de poder y de dominio de la población (la Piazza Venecia de Roma o la de Tiananmen en China). 


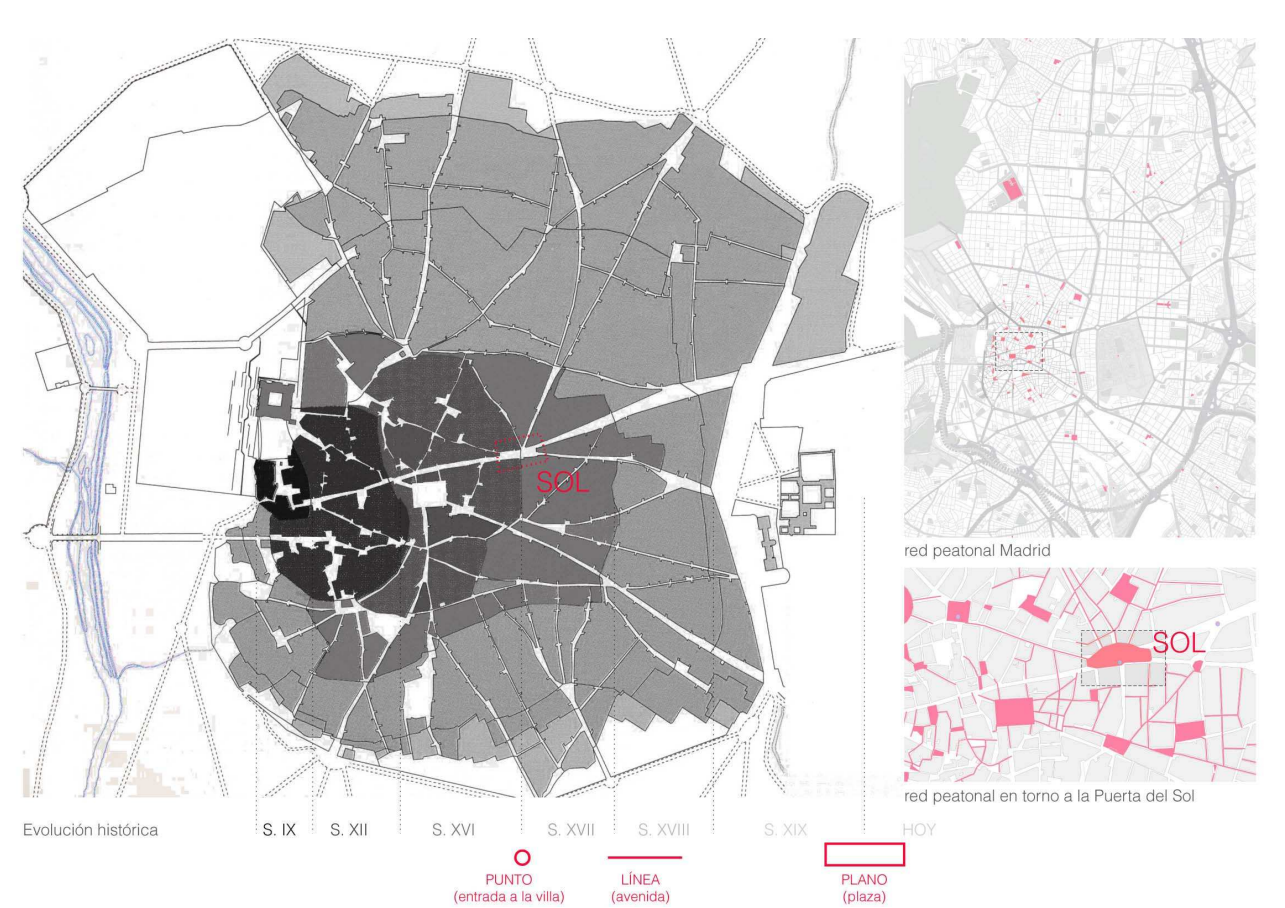

Figura 1. Puerta del Sol (en rojo) en la evolución urbana de Madrid ${ }^{16}$.

La Puerta del Sol es un espacio simbólico para los madrileños y los españoles en general. En primer lugar porque es la sede de la Presidencia de la Comunidad de Madrid, y en segundo lugar porque es además el centro geográfico del país. También es parte del tejido orgánico -con un total de diez calles convergentes en ella- de una red de espacios peatonales y plazas interconectadas que componen la zona histórica de Madrid (figura 1). Todo esto lo convirtió en el centro ideal para las protestas, no sólo simbólica e ideológicamente, sino también físicamente (todo el mundo tiene que pasar cerca de Sol, en un momento dado). Por último, destacar la idoneidad de su tamaño pequeño en relación al número de manifestantes- y su morfología rodeado de edificios. Las imágenes informativas debían, por tanto, ser tomadas desde el aire o desde la propia acampada, potenciando el efecto multitudinario -adaptándose al dictum político "realiza siempre la asamblea en una sala demasiado pequeña" (Cooper 2012).

Hasta el 15-M, la Puerta del Sol era concebida como un espacio de consumo (comercial-turistas) y de paso (nodo intercambiador de transportes). A esta percepción se une su referencia política, albergando la mencionada sede de la Presidencia de la Comunidad de Madrid. El resultado es una plaza dura, coronada por una estatua ecuestre de Carlos III, donde tiene poca cabida lo social, a pesar de que ya antes muchas manifestaciones tuviesen lugar allí, conviviendo frecuentemente con grupos de policías y vallas de protección

${ }^{16}$ Todos los mapas están realizados por las autoras del artículo. 
rodeando el edificio del Gobierno. Pero su ocupación y transformación en la primavera de 2011 le dieron una nueva dimensión y significado en la ciudad. El Movimiento 15-M, la transformación de la plaza que todos vimos durante meses en medios de todo el mundo, ha cambiado esa percepción anterior para siempre, por mucho que las fuerzas políticas se empeñen en restaurarla mediante vigilancia policial y otras medidas de seguridad, e incluso presión social ${ }^{17}$.

\section{Los efectos del Movimiento 15-M: acciones}

La ocupación de la Puerta del Sol y el Movimiento 15-M han sido fenómenos muy discutidos en los últimos dos años (Martín Patino 2012, Grueso 2012, Minguijón y Pac 2012, Jaque 2011, Taibo 2011). Sin duda la transformación física de ese espacio, la creación de una ciudad dentro de la ciudad, la movilización ciudadana por la defensa de sus derechos, merecen la atención de todo aquel que estudie lo urbano en todas sus dimensiones (económica, social, ecológica...).

También se ha de destacar el papel decisivo que jugaron las redes sociales, Internet y las nuevas tecnologías en todo el proceso. Otros movimientos, como OWS ${ }^{18}$, enmarcados dentro del mismo fenómeno, han sido muy estudiados desde el punto de vista de la ocupación del espacio público y la importancia de las redes sociales para los cambios sociales y políticos (Hugill \& Thorburn 2012; Massey \& Snyder 2012, entre otros).

La investigación presentada en este artículo -aunque toma como base los estudios de transformación física del espacio y de organización a través de las redes sociales- se centra en las nuevas acciones surgidas en el espacio de protesta (ORGANIZAR, OCUPAR, TRANSFORMAR) y en cómo incorporar ese fenómeno de acciones al estudio, diseño, gestión y uso del espacio público. No cabe duda de que se necesitan cambios. Para evolucionar y mejorar es necesario poseer un espíritu crítico como sociedad, que evalúe el modelo de ciudad que hemos venido construyendo y el contexto socio-cultural y político-económico actual.

\footnotetext{
${ }^{17}$ En junio de 2012 la entonces Presidenta de la Comunidad de Madrid, Esperanza Aguirre, otorga el Premio a la Tolerancia a los comerciantes de Sol por su "tolerancia y templanza ante la ocupación ilegal de la plaza". Llama la atención que la percepción de esa ocupación fue muy diferente en ámbitos distintos al político: Manuel Borja-Villel lo elige en Art Forum la obra de arte más importante de 2011, y se crea además una categoría especial en el Premio Europeo del Espacio Público Urbano, para reforzar su potencial (Ramírez 2012).

18 Occupy Wall Street, movimiento de protesta que surgió el 17 de septiembre de 2011 en Zuccotti Park, en el distrito financiero de Nueva York. Las protestas de OWS son un ataque a las desigualdades sociales y económicas, la codicia y la corrupción y la innegable influencia de las empresas -especialmente las financieras- sobre el gobierno.
} 
Arendt explica que la vita activa ${ }^{19}$ está compuesta por tres actividades básicas: esfuerzo, trabajo, y acción. Para este estudio interesa de forma especial la última de ellas, que está ligada al ámbito político y sólo puede tener lugar en el espacio público (según las acepciones en la antigua Grecia), para crear algo duradero. Se vale normalmente del discurso (logos), aunque algunas acciones, como la actividad en un mercado, no la requieran; y nunca es predecible y determinista en sus consecuencias, ya que el sujeto, al actuar, entra en una compleja red de relaciones que no se pueden predecir de antemano. De la misma forma, la acción es irreversible (Arendt 1958). Como veremos en el caso de la Puerta del Sol, la percepción actual que se tiene de ese espacio, tras las acciones del $15-M$, ha cambiado radicalmente en el imaginario colectivo de la ciudadanía ${ }^{20}$.

\subsection{La capacidad de auto-organización (Organizar)}

Lo que resulta interesante a día de hoy -más que los eventos que tuvieron lugar entre el $15-\mathrm{M}$ y el $12-\mathrm{J}^{21}$ - es la repercusión que el movimiento ha tenido, y cómo las ideas básicas y la organización se han trasladado a los barrios madrileños (Nez 2011), evolucionando el movimiento 15-M mediante un método de participación ciudadana, de democracia deliberativa, alternativa a la falta de acción por parte de las instituciones oficiales ${ }^{22}$. Desde la Asamblea General de Sol se crearon unas comisiones de barrios que organizan reuniones periódicas en los distintos barrios/distritos/zonas (más de 100). Estas asambleas populares o de barrio -que se basan en el modelo de organización del campamento de Sol, pero adaptándose a las realidades locales- cuentan con comités de apoyo y grupos de trabajo para proponer nuevas vías de actuación.

${ }^{19}$ El término 'vita activa', mencionado en una cita anterior, fue acuñado por Arendt y es el equivalente a la libre acción política del ciudadano de la antigua polis griega.

${ }^{20}$ La práctica de la 'ciudadanía' en la esfera social ha ido ganando preponderancia en las ciudades europeas. La creciente responsabilidad de las instituciones locales en la política social requiere nuevas prácticas institucionales para poder incorporar más cantidad de actores que sean más heterogéneos (García 2010).

${ }^{21}$ Intervalo de tiempo que duró la acampada de la Puerta del Sol, del 15 de mayo al 12 de junio de 2011.

22 Creación de nuevas plataformas digitales y blogs, como: http://madrid.tomalaplaza.net/, http://madrid.tomalosbarrios.net/, http://papers.15m.cc (recopilación de artículos sobre el movimiento 15-M). Se utilizan como fuente de los eventos ocurridos para esta investigación: Algaba Calderón 2012, Cabrera Viñas 2012, Callejo 2012, Martínez Galera 2012. 
El movimiento de los indignados ${ }^{23}$ desarrolla técnicas establecidas de deliberación y auto-organización. La creación de órganos autogestionados en distintos barrios a raíz del 15-M -creados por los mismos medios que los de Lavapiés ${ }^{24}$ en los $90-$ ha potenciado la aparición de nuevos centros de barrios, donde verdaderamente se practica la dimensión social y democrática de la ciudad. Este tipo de participación directa que ahora parece natural, en los años 90 era aún rara y rozaba los márgenes de la normalidad/legalidad.

Por primera vez en la historia, gracias a internet, los eventos y la información viajan de forma simultánea a cualquier parte del planeta. Si para la creación de órganos sociales autogestionados en el barrio de Lavapiés en los años 90 hicieron falta años de diálogo con el Ayuntamiento, puesta en común de los vecinos, etc., con la rapidez e inmediatez con las que contamos hoy en día gracias al poder de convocatoria de las redes sociales, se pudo auto-organizar el espacio de Sol, tanto física como funcionalmente, en pocas horas. Los avances tecnológicos fueron un medio, pero lo verdaderamente relevante como fenómeno urbano fue la auto-organización en sí de los ciudadanos, su capacidad para hacer ciudad y promover cambios y adaptación. Sin duda este último aspecto es lo que determina el carácter e identidad de una ciudad, la diferencia que Jane Jacobs apuntaba entre las ciudades vivas - capaces de regenerarse y autoinventarse- y aquéllas enfermas o muertas -que sólo contribuyen a su autodestrucción ${ }^{25}$.

\subsection{El fenómeno de ocupación del espacio público (Ocupar)}

El espacio no es "reflejo de la sociedad", es la sociedad [...]. Por lo tanto, las formas espaciales, por lo menos en nuestro planeta, se producirán, como todos los demás objetos, mediante la acción humana. Expresarán y representarán los intereses de la clase dominante, de acuerdo con un determinado modo de producción y de

${ }^{23}$ El término con el que se definen hace referencia al título del libro Indignez-vous! (iIndignaos!) escrito por el diplomático francés Stéphane Hessel, redactor en 1948 de la Declaración de Derechos Humanos. Castells, en su nuevo libro Networks of Outrage and Hope: Social Movements in the Internet Age (2012), habla sobre el movimiento de los indignados, que ocurrió cuando nadie lo esperaba, y analiza el contexto en el que ocurrió (crisis económica, cinismo político, vaciedad cultural y desesperanza), identificando nuevas vías para el cambio social en este siglo y planteando hipótesis sobre sus implicaciones en la ciudad.

24 El caso de Lavapiés, los Centros Sociales Autogestionados y/o Ocupados (CSA/Os, Vidania 2012). Es un antecedente interesante de lo que ha ocurrido en los barrios, a raíz del Movimiento 15-M. Lavapiés ha experimentado un proceso de rehabilitación desde el año 1996 . Tiene una dinámica singular de autorregulación del barrio.

25 "Las ciudades vitales tienen su propia semilla de regeneración para el futuro, mientras que las inertes e impersonales tienen la de su destrucción" (Jacobs 1961). 
desarrollo. Expresarán e impondrán las relaciones de poder del Estado en una sociedad históricamente definida [...]; de vez en cuando, los movimientos sociales se levantarán para desafiar el sentido de la estructura espacial y por lo tanto, ensayar nuevas funciones y nuevas formas. (Castells 1983: 4)

Gracias a las redes sociales, la libertad que muchas veces se ve coartada por políticos, leyes y fuerzas de seguridad, queda libre de restricciones para poder organizarse, movilizarse e intentar transformar y cambiar tanto el espacio físico como el plano legal ${ }^{26}$. Castells (2012) analiza la capacidad que las personas, conectadas a través de las redes sociales de internet, tuvieron para agruparse en esos espacios de autonomía -en este caso la Puerta del Sol- y, desde la "seguridad virtual", ocuparon el espacio público y elaboraron iniciativas ciudadanas ligadas a sus verdaderas preocupaciones. En todos los casos, dice Castells, ignoraron a los partidos políticos en el poder, desconfiaron de los medios de comunicación y, rechazando las formas de liderazgo y de organización formales, debatieron colectivamente, tomando sus decisiones, como vimos en el punto anterior, en asambleas locales y a través de internet.

La palabra ocupar en el contexto que estudiamos tiene dos acepciones válidas: "tomar posesión o apoderarse de un territorio, de un lugar, de un edificio, etc., invadiéndolo o instalándose en él", y "llenar un espacio o lugar". Aunque convendría reflexionar acerca de esta otra: "llamar la atención de alguien; darle en qué pensar". Los ciudadanos madrileños no sólo tomaron posesión de un espacio, llenándolo de personas, en el centro neurálgico de la capital, sino que -y en parte gracias a los nuevos medios de comunicación- llamaron la atención de muchas otras personas no presentes allí; entre ellas, los políticos, que vieron la ocupación como una amenaza. Como defiende Lefevbre (1991) en sus textos, la ocupación del espacio ha sido siempre un símbolo de poder y, por ello, las fuerzas en el poder siempre han procurado controlarlo.

El fenómeno de ocupación está íntimamente ligado con la regeneración del espacio físico y social. Esa conquista de la ciudad no es sólo un símbolo de poder, sino que además representa algo extraordinario: la salida de los ciudadanos de su ámbito privado (la vivienda, el lugar de trabajo, etc.) al ámbito público, para desarrollar sus actividades y hacerse oír, y de esta forma, no sólo reivindicar sus derechos, sino activar y regenerar el propio espacio público,

26 Destacan especialmente las revueltas de la Primavera Árabe, cuando manifestantes egipcios, sirios, etc., gracias a la infraestructura de las nuevas tecnologías, ocuparon las principales plazas de sus ciudades para protestar por sus gobiernos, en países donde previamente no existía el derecho a la libertad de expresión y a las manifestaciones. 
dotándolo de una nueva dimensión, más acorde con la ciudadanía en su conjunto y con el momento actual.

\subsection{El poder de transformación (Transformar)}

A través de acampadas, la producción y exposición de carteles de protestas, la creación de bibliotecas y puestos temporales para la comunicación y difusión, la recolección y preparación de alimentos, el diálogo con los transeúntes, y la participación en una toma de decisiones compartida, los manifestantes transformaron una zona urbana [Zucotti Park, vid. n. 17] normalmente pasiva y mundana, en un lugar de expresiones políticas y acciones colectivas activas. (Hou 2012: 92)

La transformación de la Puerta del Sol (junto con otros espacios ligados al movimiento de ocupación), pone de manifiesto la capacidad de los ciudadanos, de cada individuo, para moldear la estructura diaria de una ciudad. Lo interesante no fueron sólo las movilizaciones de los indignados y cómo transformaron la Puerta del Sol, sino la verdadera transformación de lo "público" en el espacio público. El término público tiene tres significados principales: 1) conjunto de personas que concurren un mismo lugar, 2 ) lo perteneciente a todo el pueblo o ciudad y 3) lo notorio, patente, manifiesto, visto o sabido por todos. Hou (2012) sugiere que el término público no es ya sólo un adjetivo del diccionario, sino que para el marco de esta investigación se puede entender como un cuerpo activo de ciudadanos (active body of citizens) en un sentido más amplio de la palabra. La ocupación de Sol fue ante todo un acto de la producción y protección de lo público, tanto física como políticamente; la transformación de un espacio urbano en un lugar de acciones, de posibilidades y de significado. Se destaca por tanto lo público como cuerpo activo de ciudadanos -con capacidad transformadora y de acción- frente a lo público como espacio físico y formal, determinado por leyes, con capacidad de representación ${ }^{27}$.

27 Ya la vida griega se dividía en dos mundos: el de lo público, en el que se encuentra la actividad política, y el de lo privado. Desde los romanos, un tercer mundo ha ido apareciendo: el social. La esfera pública es el ámbito donde se ejerce una verdadera libertad adquirida, donde los ciudadanos debaten sobre temas más allá de la mera vida, desprovisto de intereses personales o privados (asuntos públicos como la educación, la guerra, el derecho, etc., que afectan a todos los ciudadanos). La violencia está totalmente excluida de este ámbito, donde el éxito viene de la propia persuasión de los demás mediante la razón y el poder retórico (Arendt 1958). 


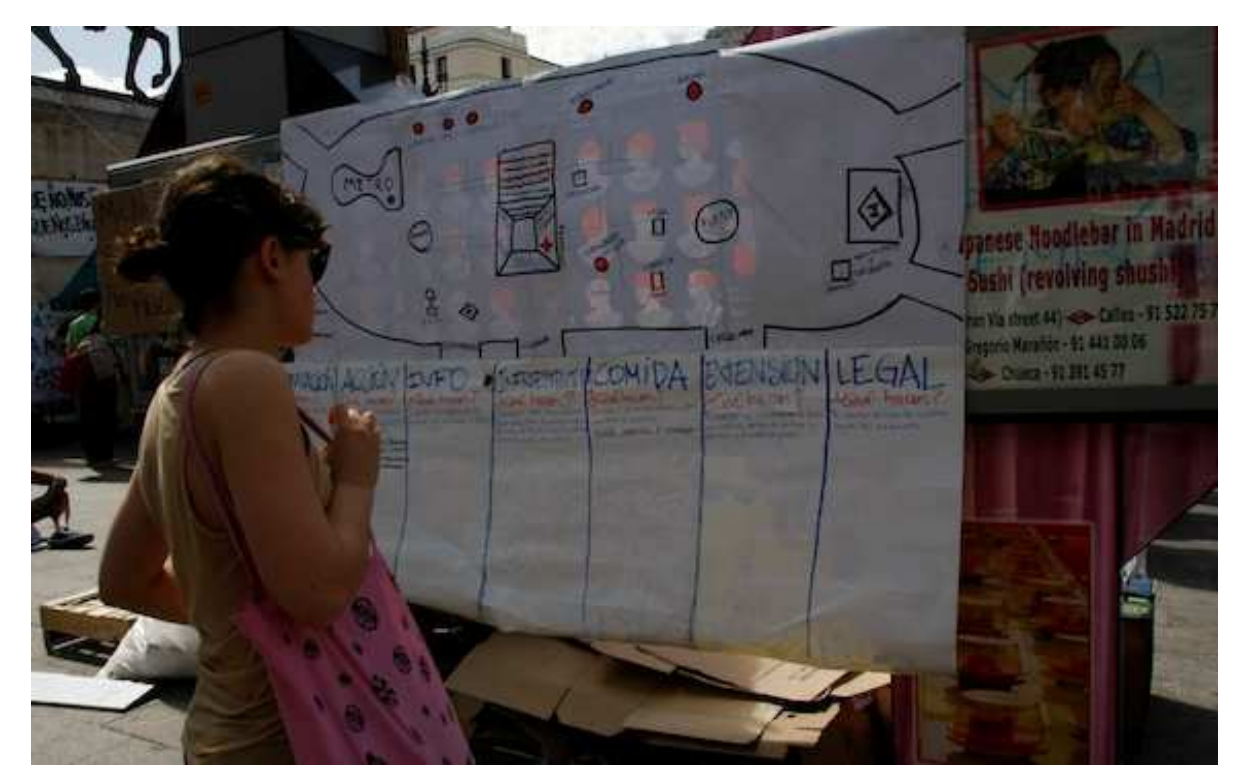

Figura 2. Mapa en la Puerta del Sol creado durante la Acampada en $2011^{28}$.

Como se aprecia en la figura 2, los ciudadanos que ocuparon el espacio de Sol realizaron mapas, en los que se señalaba cómo organizar la circulación, dónde localizar funciones y usos, etc. La representación de la forma de la plaza va acompañada de palabras, de estrategias sobre cómo transformar y organizar ese espacio. En definitiva, crearon acciones $-y$ las mapearon- sobre un espacio que hasta entonces había sido sólo para representaciones, y así transformaron completamente, y seguramente de forma poco consciente, no sólo su imagen y su espacio físico, sino también su significado en la ciudad.

Jeffrey Hou en su libro Espacio Público Insurgente (Insurgent Public Space, 2010) hace una distinción entre el espacio público institucional y el insurgente ${ }^{29}$. El primero de ellos hace referencia a los parques urbanos, plazas, calles, edificios públicos, espacios públicos gestionados de forma privada, etc. Estos están regulados, normalizados, mantenidos y gestionados por las instituciones. El segundo tipo, insurgente, engloba los espacios creados por los ciudadanos, en ocasiones fuera del margen de la legalidad. En el desarrollo de la investigación se estudian ambos tipos de espacios en el contexto madrileño: el permanente y regulado por leyes y ordenanzas (1) frente al insurgente y a veces efímero, creado a raíz de movimientos sociales y espontáneos (2).

${ }^{28}$ Todas las fotografías han sido tomadas por las autoras del artículo.

29 José Ángel Bergua Amores en su libro Lo social instituyente: materiales para una sociología no clásica ya analizaba las relaciones entre lo instituido y lo instituyente en 2007, haciendo hincapié en la fuerza de los ciudadanos (lo instituyente). 


\section{Lo espontáneo se abre paso por lo institucional \\ 4.1. El marco legal}

Los órganos de poder determinan qué partes de la ciudad son más visibles y qué se puede decir acerca de ellas $[. .$.$] , construyen$ determinadas narrativas de la ciudad en la economía simbólica e influyen en la valoración de un lugar: dónde ha de ser invertido el capital, qué lugares se han de comercializar, y qué políticas han de ser promulgadas. El poder de estos imaginarios radica en su capacidad para fomentar algunos lugares en el mapa cognitivo de los ciudadanos. (Rios 2012: 135)

El espacio público, aún estando gestionado y mantenido por, entre otros agentes, el ayuntamiento, es tratado muchas veces de forma heterogénea en la totalidad de la ciudad. Un plan general, la realización de un plan parcial de un tipo u otro, las ordenanzas y los recortes presupuestarios (tan característicos de estos tiempos de crisis), tienen una influencia directa en la mejora o el deterioro del paisaje urbano ( $v i d$. figura 3 ).

El urbanismo, como se ha venido ejerciendo en España, facilita y permite a las fuerzas del poder (políticos) visionar, planear y regular la ciudad. No tiene en cuenta un equilibrio social y nuevas dimensiones en el espacio público, como lugar también de acciones y no sólo de representaciones (como los espacios de consumo del neoliberalismo ${ }^{30}$ ). "La ciudad representa nuestro modelo político y económico" (Lefebvre 1991), al cuál urge añadir el modelo social. "Para reproducir control social, el Estado debe reproducir control espacial" (Deleuze y Guattari apud Soja 2000: 361).

En las últimas décadas, el ámbito de la intervención social, al igual que el de la educación o la sanidad, ha dado lugar progresivamente a formas de gestión público-privada dominadas por la "lógica de mercado". El planeamiento y el diseño urbano no han sabido incorporar temas sociales al espacio público, ya que en ocasiones centran su atención en lo estético y formal, dejando poco espacio para las cuestiones éticas, sociales y políticas que surgen desde el ámbito público, en constante cambio y evolución.

El espacio público está poco regulado en algunos ámbitos (lagunas en el planeamiento urbano) y demasiado regulado en otros (exceso de diseño urbano). Existen en nuestro país vacíos sobre cómo debe ser regulado y gestionado el espacio público, otorgando a los políticos un excesivo poder sobre el espacio público mediante ordenanzas y normas ciudadanas, cuando en realidad éstas deberían

30 El mejor ejemplo es el de los grandes centros comerciales, ubicados en la periferia de nuestras ciudades, que crean una micro-ciudad comercial, artificial, basada fundamentalmente en el consumismo, en lugar de en la interacción social (excepción: los malls americanos, convertidos en lugares sociales para los teenagers). 
decidirse de forma más deliberada y democrática. La ocupación de un espacio para el uso común está controlada por normas que muchas veces no son claramente conocidas, reconocibles o racionales.

En cambio, el espacio público está demasiado regulado en cuanto a estética, forma, función, etc. Basado en un sistema rígido, que por una herencia cultural y de normas de propiedad y hábitos de consumo (comercio) dicta cómo se debe usar ese espacio (para la representación y no la acción), qué actividades se pueden o no realizar en él, etc. El espacio queda entonces sobreprogramado de una manera rígida, dejando poco lugar a lo espontáneo (como el caso de Sol, vid. figura 5, mapa izqda.).

El principio esencial de la democracia radica en la capacidad de los ciudadanos de guiar la dirección de las políticas públicas ${ }^{31}$. Los ciudadanos se sienten impotentes ante las medidas adoptadas por los políticos en general, y ante las leyes que "controlan" el espacio público, las asentadas y las manifestaciones. ¿Cómo de público es verdaderamente el espacio público?

\subsection{Los movimientos sociales}

En el conglomerado de debates, iniciativas y protestas englobadas bajo la denominación $15-\mathrm{M}$ ha habido reivindicaciones razonables. [...] tiene en su haber un triunfo indudable y meritorio, haber llamado la atención de los grandes partidos y a que se apunten cambios de la legislación injusta [sobre los desahucios]: una prueba de que con objetivos y decisión crítica la transformación social no es una mera utopía. (Savater 2012)

El escenario en el que ha hecho su aparición la crisis y, con ella, movimientos ciudadanos como el $15-\mathrm{M}$ es un panorama urbano y social desolador, íntimamente relacionado con un modelo de ciudad y unas decisiones socio-económicas, financieras y políticas llevadas a cabo en la última década. Tres factores principales han de tenerse en cuenta. El primero, la falta de espacios públicos de reunión, foro y debate, en áreas planificadas modernistas ${ }^{32}$, donde las personas no se sienten representadas ${ }^{33}$. El segundo, la actual Ley del Suelo y los

${ }^{31}$ El indio Amartya Sen (Nobel de Economía) ha defendido que la democracia, más allá de la representación política y del respeto a la regla de la mayoría, implica la protección de los derechos y las libertades de los individuos, el acceso a las prestaciones sociales y el derecho a acceder a la información, así como de participar activamente en la deliberación colectiva.

32 Ejemplo paradigmático de este nuevo modelo urbano es el de los nuevos barrios de Madrid, los PAUs, conjunto de ambiciosas operaciones en la periferia del municipio que han agotado el suelo vacante con la construcción de 200.000 nuevas viviendas (para 500.000 personas), sin apenas espacios públicos comunes.

${ }^{33}$ Han pasado ya décadas desde que se extendió el modelo de ciudad funcional por todo el mundo, pero pocos estudios analizan los efectos de esta perfecta sociedad de propietarios, y la pobreza de una ecología social que se limita al trabajo, al ocio 
Planes Generales madrileños, utilizados como herramientas económicas ${ }^{34}$, en lugar de actuar como garantía social que beneficiase a la totalidad de los ciudadanos. Esta colonización del territorio madrileño (principalmente para el uso de vivienda) no ha venido sola. El tercer y último factor para la situación de crisis urbana y social madrileña ha sido el desarrollo y financiación de grandes infraestructuras de comunicación ${ }^{35}$.

"La burbuja inmobiliaria ha sido durante muchos años el anestésico que tenía dormida a la profesión y hacía que ni colectivos, ni redes, ni nuevos formatos emergieran con la fuerza con la que lo han hecho" (Villegas 2012). El término anglosajón doldrums (periodo de inactividad) define perfectamente ese estado de somnolencia de participación ciudadana en la que la mayoría de madrileños permanecíamos sumidos desde las revueltas urbanas de los sesenta y setenta por el "derecho a la ciudad". El sociólogo y psicólogo Habermas es uno de los estudiosos más optimistas sobre la reactivación de la esfera pública. El camino para ello, dice, pasa por sustituir la democracia representativa -dependiente del Estadonación- por una democracia deliberativa -organismo político basado en la igualdad de derechos y obligaciones de los ciudadanos. En este sistema de democracia directa son importantes tanto el activismo para los debates sobre asuntos de importancia pública, como el mecanismo para que ese debate pueda tener alguna repercusión en el proceso de toma de decisiones (Habermas 1997).

La situación entonces era más precaria, pero hoy en día hay una creciente fractura social, que no se limita simplemente al empleo. Aparecen nuevas "especies sociales" 36 asociadas a lo que nos gusta llamar, en esta investigación, especies de espacios. Las distintas especies de espacio público tienen, por tanto, distinto uso, distintas formas de relación social y distintos hábitos de consumo ${ }^{37}$. Daniel Innerarity ahonda en esta idea de espacio público y sus

dirigido y a la vida privada en el interior de una vivienda tan rígidamente separada de la ciudad.

34 Durante toda una década (1993-2003) el uso intensivo del territorio ha sido el factor clave del crecimiento económico madrileño, aumentando el desarrollo urbanístico un $49 \%$ (cantidad equivalente a la mitad de lo que ha crecido Madrid en toda su historia). Un gran número de municipios de la Comunidad ha duplicado sus dimensiones y algunos han multiplicado su tamaño hasta diez veces.

35 Todas ellas sufragadas con dinero público -e incluso europeo-. Sin duda todo un progreso, que ha convertido a Madrid en la ciudad europea con mayor número de kilómetros de autovía por millón de habitantes (un total de 1.000).

36 Término extraído del manifiesto "Madrid, ciudad global" (p. 23).

37 El nuevo modelo se ha basado en una acelerada destrucción de los bienes "comunes", como la sanidad, la educación y el territorio. En algunas zonas o barrios, se recurre cada vez más a iniciativas sociales autogestionadas, como el 15-M para buscar una igualdad territorial y urbana, y surtir de usos básicos e infraestructuras el espacio público. 
transformaciones en la sociedad contemporánea. Analiza los actuales escenarios en los que se desarrolla la vida pública, y propone una transformación del poder político de manera que esté en condiciones de articular espacios comunes, cooperación, responsabilidad e integración en la sociedad mundial contemporánea (Innerarity 2006).
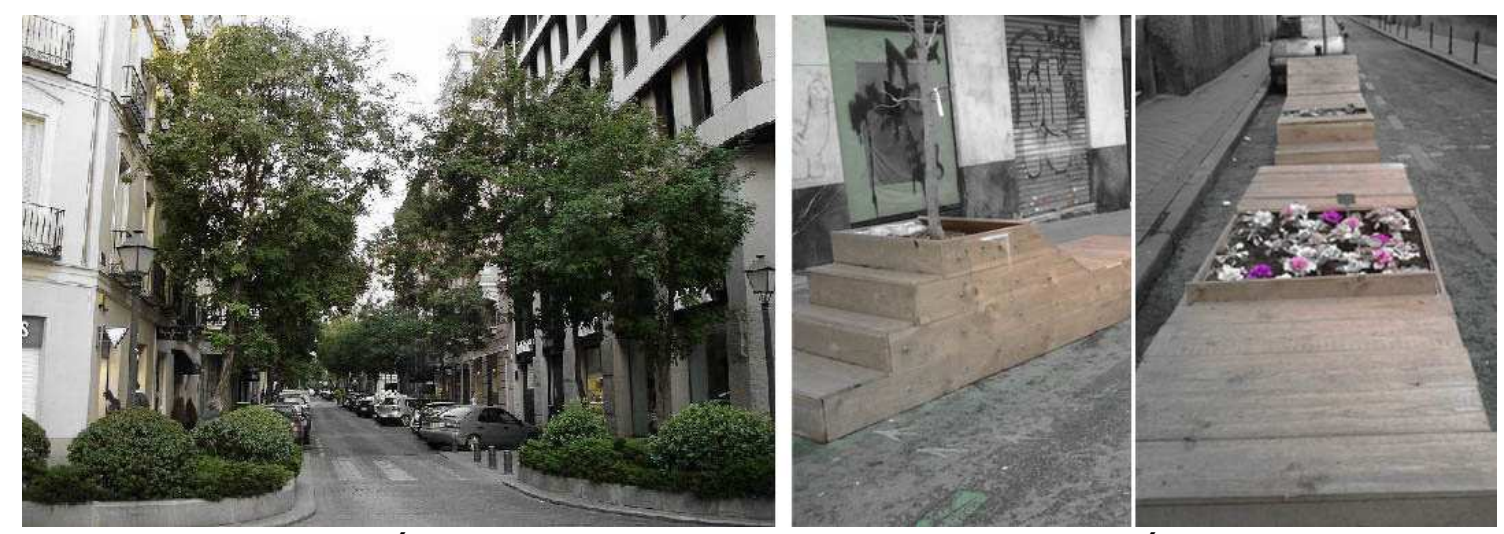

Figura 3. Vegetación urbana diseñada por la administración en el barrio de Salamanca (izqda.), frente al mobiliario urbano diseñado por grupos vecinales en el barrio de La Latina (dcha.).

A parte del fenómeno social del Movimiento $15-\mathrm{M}^{38}$ hay muchas otras iniciativas repartidas por todo el territorio madrileño, a todas las escalas, como Tabacalera ${ }^{39}$, La Plaza de la Cebada ${ }^{40}$, "cuando hay un solar, hay un huerto!", etc. La ciudadanía esta creando nuevos modelos de gestión. Si ilegalmente se pueden conseguir cambios tan beneficiosos para la ciudadanía, si los vecinos de un barrio son capaces de transformar su espacio a mejor (figura 3, imagen de la derecha), sin necesidad de leyes u ordenanzas, ¿no deberíamos replantearnos hasta dónde llega lo regulado y qué y cómo regularlo?

${ }^{38}$ Leonard Buff escribe en el Foro Social de Porto Alegre en 2012 cómo los ideales y críticas del "movimiento de los indignados madrileño" o "movimiento 15-M" es hoy la mejor alternativa de vanguardia para dar respuesta a la situación política y económica de la crisis económica mundial que sufrimos desde 2008.

39 Hace dos años, el Ministerio de Vivienda acogió la idea de Centro Social Autogestionado (CSA) como recurso público, sin ningún tipo de condiciones: "Tabacalera a debate". Fue una cesión. Es la primera vez que en Madrid se consigue un volumen de estas características para ser gestionado por la ciudadanía. ${ }^{40}$ Intervención ciudadana participativa madrileña (http://elcampodecebada.org/). Los vecinos reclaman el derecho a un centro deportivo después de la demolición de un complejo deportivo y de la retirada del proyecto, dejando una parcela vacía en el centro. Los ciudadanos tomaron el espacio y en la actualidad se organizan diferentes actividades sociales y culturales, transformando el espacio y demostrando que ese uso es muy necesario en esa área. 


\section{Conclusiones: la fina barrera entre lo público y lo privado, lo legal y lo ilegal, lo institucional y lo ciudadano}

Las ciudades son los instrumentos más avanzados de lo que Michele Foucault denomina biopoder, el poder de hacer vivir y dejar morir. En el mapa de la ciudad es donde se puede ver de forma más real que la gobernabilidad no es una cuestión de imponer la ley, sino de regular las cosas para que la conducta individual se sitúe dentro de un marco determinado de acciones posibles. (Segal 2012: 157)


CONMUTAR / CONSUMIR / DESFILAR / VIGILAR / CIRCULAR / ESPERAR OCUPAR / ORGANIZAR / TRANSFORMAR / HABITAR/ INFORMAR / DIALOGAR

Figura 4. Esquema de la Puerta del Sol como espacio institucional (izqda.) y como espacio insurgente (dcha.). En el primero, el rojo corresponde a la principal actividad de la zona, el comercio. En el segundo, el rojo indica las zonas de acción donde se crearon todo tipo de actividades sociales durante la acampada del $15-\mathrm{M}^{41}$.

En los mapas de la figura 4 quedan reflejados los resultados de la investigación: la dualidad de los espacios contemporáneos; con carácter institucional y actividades de representación (figura 4, izqda.) y con carácter insurgente y actividades de acción (figura 4, dcha.). Mientras que la característica primera es fruto de las leyes y de la gestión privada y de la administración, la segunda es fruto de la cooperación ciudadana, de un sistema deliberativo y transparente y de la autogestión. Mientras que el primero surge de la representación fruto de los usos implantados en los bajos comerciales de los edificios que rodean la plaza, el segundo surge de la acción ciudadana, de sus ideas y de sus actos. ¿Cómo podemos mirar más allá del Movimiento 15-M y la ocupación de Sol? ¿Cómo hacer que este tipo de procesos repercutan no sólo en distintos barrios madrileños gracias a la autogestión del propio movimiento, sino en el sistema en general, en el marco legal y en el ámbito del urbanismo, de hacer ciudad y en la labor de crear, diseñar y gestionar el espacio público?

41 Mapas realizados por las autoras del artículo a partir de datos obtenidos de Acampadasol. 
Para ello es clave tomar como punto de partida que la creación de lo social y lo político ocurre mediante y gracias a la participación y creación ciudadana, como una práctica cotidiana que debe incluir un mayor número de actores y procesos. A continuación se exponen unas posibles directrices a seguir, conclusiones de la investigación aquí presentada:

Visionar: El espacio público debe ser imaginado por más actores, por el colectivo ciudadano. Todos vamos a habitar ese espacio, y por tanto no puede ser sólo el "sueño" de un político o un arquitecto.

Planear: La planificación debe ser flexible y no rígida, anticipando el futuro y adaptándose a todo tipo de ocasiones y condiciones (el bello término anglosajón constrains). La rigidez de las barreras en la ciudad, tanto físicas como sociales, debe ser cuestionada. Se deben crear relaciones híbridas, más fluidas entre lo público y lo privado. Muchos no-lugares o terrain vagues, indicadores del deterioro o abandono de un barrio, se utilizan finalmente por los vecinos locales para realizar actividades diferentes de aquellas para las que fueron diseñados o designados legalmente ${ }^{42}$.

Diseñar: Se debe potenciar el placemaking ${ }^{43}$ y no lo puramente estético (frivolidad del diseño por el diseño) o lo permanente (ya superado). La habilidad de los arquitectos y urbanistas para producir una imagen inclusiva y equilibrada de la ciudad está íntimamente relacionada con la capacidad que tengan de hacer que la gente se imagine su espacio público y se sienta representada en él. El espacio debe ser diseñado según una serie de capas que varían en función de los distintos escenarios, dotándolo de flexibilidad.

Gestionar: Introducir las acciones y no sólo las representaciones. El espacio público no se vincula sólo al comercio y la restauración (terrazas) o al transporte, etc. Dado que la gestión normalmente va asociada al agente financiador, el verdadero reto para arquitectos y urbanistas es crear funcionalidad e integración ciudadana, con o sin financiación del gobierno. Las favelas de São Paulo, así como muchas otras ciudades, nunca han sido financiadas por infraestructura municipal. ¿Fue la acampada de Sol un tipo de favela, al borde de lo ilegal, en el borde de una ciudad próspera y polarizada? Una ciudad verdaderamente pública y justa es aquélla en la que existe un equilibrio y un tamiz social homogéneo, y no aquélla

\footnotetext{
42 "596 acres" en Estados Unidos, "esto no es un solar" en Zaragoza, "cesión de espacios a la ciudadanía" en Euskadi, Casa Caracol y Tabacalera en Madrid, etc.

43 Término anglosajón sin traducción al castellano, que hace referencia al acto de promover que ocurran cosas en un lugar, subraya formas novedosas mediante las que los "marginados" o "indignados" pueden desarrollar su cultura o identidad política, reclamando no sólo el espacio físico sino también sus derechos.
} 
llena de islotes o fronteras, como en las favelas de Rio. Las tácticas ${ }^{44}$ o estrategias ${ }^{45}$-que no estratagemas ${ }^{46}$ - para propiciar un espacio público inclusivo y homogéneo en toda la ciudad son cruciales.

Regular / Negociar: Las ordenanzas no deben estar sólo a disposición de los propietarios, políticos y demás fuerzas del poder, sino que deben ser por y para el bien ciudadano, y desarrolladas entre todos. Doreen Massey habla de cómo los lugares nos cambian, no por un sentimiento visceral de pertenencia, sino por la práctica o el practicing de un lugar, entendiendo éste como la arena donde la negociación se puede llevar a cabo (Massey 2005).

Los arquitectos y urbanistas deben ser brokers culturales y actuar de charnela entre los movimientos sociales y las agencias gubernamentales, las instituciones y los intereses privados. Como profesionales creamos ese equilibrio entre lo institucional y lo espontáneo, lo social y lo, en ocasiones, ilegal. Para que nuestras ciudades sean, como lo han sido desde tiempos inmemoriales, el resultado del desarrollo humano, debemos procurar además no sólo las acciones colectivas sino también las de cada ciudadano, promoviendo así la cohesión social, ese intermediario entre lo institucional y lo insurgente, lo representativo y la acción, para conseguir un mayor equilibrio e inteligente diseño y utilización del espacio público urbano.

44 1. Método o sistema para ejecutar o conseguir algo. 2. Conjunto de reglas a las que se ajustan en su ejecución las operaciones militares (DRAE). Michel de Certau (1984: 34) describe las tácticas (tactics) como "las corrientes en un mar teóricamente gobernado por un marco institucional que gradualmente se va erosionando y desplegando".

45 1. Arte de dirigir las operaciones militares. 2. Arte, traza para dirigir un asunto. 3. En un proceso regulable, el conjunto de las reglas que aseguran una decisión óptima en cada momento (DRAE).

${ }^{46}$ 1. Ardid de guerra. 2. Astucia, fingimiento y engaño artificioso (DRAE). 


\section{Bibliografía}

ALGABA CALDERÓN, Fernando (2012): "La Spagna in piazza. Yes, we camp" [en línea], en papers.15M.cc, 06/08/2012. En: http://papers.15m.cc/p/listado-de-papers.html [Consulta: diciembre de 2012].

ARENDT, Hannah (1958): The Human Condition. Chicago: University of Chicago Press.

BERGUA AMORES, José Ángel (2007): Lo social instituyente: materiales para una sociología no clásica. Zaragoza: Prensas Universitarias de Zaragoza.

CABRERA VIÑAS, Josu (2012): "Naturalesa i organització de les actituds" [en línea], en papers.15M.CC, 06/08/2012. En: http://papers.15m.cc/p/listado-de-papers.html [Consulta: diciembre de 2012].

CALLEJO, Ana C. (2012): "\#15M: De Twitter a la plaza" [en línea], en papers.15M.cc, 13/08/2012. En: http://papers.15m.cc/p/listado-depapers.html [Consulta: diciembre de 2012].

CASTELLS, Manuel (1983): The City and the Grass Roots. California: University of California Press.

- (2012): Networks of Outrage and Hope: Social Movements in the Internet Age. Cambridge: Polity Press.

CCEIM: Centro Complutense de Educación e Información Medioambiental [en línea]. En: http://www.ucm.es/info/fgu/pensamiento/cceim/index cceim.php [Consulta: diciembre de 2012].

Centro Social Autogestionado en la antigua fabrica de tabacos de Lavapies [en línea]. En: http://latabacalera.net/ [Consulta: diciembre de 2012].

COOPER, Alexander (2012): "Places that matter", en Rick Bell et al. (eds.), Beyond Zuccotti Park, pp. 207-213. Oakland, California: New Village Press.

DE CERTAU, Michel (1984): The practice of everyday life. Berkeley: University of California Press.

FORESTER, John (1999): The Deliberative Practitioner. Cambridge, Massachusetts: MIT Press.

GARCÍA, Marisol (2010): "Prácticas de ciudadanía y gobernanza en las ciudades". Miríada, año 3, núm. 6, pp. 119-153.

GEHL, Jan (2010): Cities for People. Washington DC: Island Press.

GINSBORG, Paul (2008): Democracy: crisis and renewal. London: Profile Books LTD.

GRUESO, Stéphane (2012): "15M movimientos de ocupación, la expresión colectiva del descontento". TEDxBuenosAires 2012 (Teatro San Martín, 21 de mayo de 2012), TEDx.

- (2012): Excelente, revulsivo, importante. España: madrid15m.cc.

HABERMAS, Jürgen (1997): The structural transformation of the public sphere. Boston: MIT Press.

HATZISTEFANOU, Aris; \& KITIDI, Katerina (2012): Catastroika. Grecia: Infowar. 
HERRERO LÓPEZ, Yayo (2012): "Cohesión social", en Agustín Hernández Aja (coord.), Re-hab, regeneración urbana integrada (Madrid, 28-30 octubre de 2012). Madrid: ETSAM.

HOU, Jeffrey (2010): Insurgent Public Space: Guerrilla Urbanism and the Remaking of Contemporary Cities. USA: Routledge.

- (2012): "Making Public, Beyond Public Space", en Rick Bell et al. (eds.), Beyond Zuccotti Park, pp. 156-169. Oakland California: New Village Press.

HUGILL, David; \& THORBURN, Elise (2012): "Interview with 'Bifo': Reactivating the Social Body in Insurrectionary Times". The Berkeley Planning Journal, "New Spaces of Insurgency", vol. 25, issue 1, pp. 210-220.

INNERARITY, Daniel (2006): El nuevo espacio público. Madrid: Espasa.

JACOBS, Jane (1961): The Death and Life of Great American Cities. UK: Modern Library.

JAQUE, Andrés (2011): "15M and YES WE CAMP!" [en línea]. Domus, 20/07/2011. En: www.domusweb.it/en/op-ed/15m-and-yes-wecamp-controversy-as-urbanism/ [Consulta: diciembre de 2012].

LEFEBVRE, Henri (1991): The Production of Space. Cambridge: Blackwell.

MARTÍN PATINO, Basilio (2012): 15-M, libre te quiero. Madrid: Martín Patino.

MARTÍNEZ GALERA, Ana (2012): "Ensayo sobre el colectivo 15M" [en línea], en papers.15M.cc, 05/08/2012. En: http://papers.15m.cc/p/listadode-papers.html [Consulta: diciembre de 2012].

MASSEY, Doreen (2005): For Space. London: Sage.

MASSEY, Jonathan; \& SNYDER, Brett (2012): "Occupying Wall Street: Places and Spaces of Political Action" [en línea]. The Design Observer Group, 17/09/2012, vol. Forum of Design for the Public Realm. En: http://places.designobserver.com/feature/occupy-wall-street-placesand-spaces-of-political-action/35938/ [Consulta: diciembre de 2012].

MINGUIJÓN, Jaime; y PAC, David (2012): "15M. Una explicación en clave sociológica". Prisma Social, revista de ciencias sociales, núm. 8, pp. 414-439.

NEZ, Héloïse (2011): "Le mouvement des indignés s'ancre dans les quartiers de Madrid" [en línea]. Metropolitiques.eu, Volumen Terrains. En: http://www. metropolitiques.eu/Le-mouvement-des-indignes-sancre.html [Consulta: diciembre de 2012].

RAMIREZ, Julia (2012): "Urbanismo de Revuelta". Arquitectura Viva, núm. 145, "Colectivos Españoles", p. 112.

RIOS, Michael (2012): "Emplacing Democratic Design", en Rick Bell et al. (eds.), Beyond Zuccotti Park, pp. 133-140. Oakland, California: New Village Press.

ROMANOS, Eduardo (2012): "Esta revolución es muy copyleft; Entrevista a Stéphane M. Grueso a propósito del 15M". Interface, a Journal for and about social movements, núm. 4, vol. 1, pp. 183-206.

SASSEN, Saskia (2011): "The Global Street: Making the Political". Globalizations, vol. 8, núm. 5, pp. 565-571.

SAVATER, Fernando (2012): "Un triunfo del 15-M". El País, 20/11/2012. 
SEGAL, Paula Z. (2012): "Room to grow something", en Rick Bell et al. (eds.), Beyond Zuccotti Park, pp. 156-169. Oakland, California: New Village Press.

SOJA, Edward (2000): Postmetropolis: Critical Studies of Cities and Regions. USA: Blackwell Publishing.

TAIBO, Carlos (2011): "Sobre el programa del Movimiento 15-M" [en línea], en Globalízate.

En:

http://globalizate.org/getArticle?authors=Carlos+Taibo\&date $=2011$ 06-05\&title=Sobre+el+programa+del+movimiento+15-M [Consulta: diciembre de 2012].

Toma los barrios / Asamblea Popular de Madrid [en línea]. En: http://madrid.tomalosbarrios.net/ [Consulta: diciembre de 2012].

VIDANIA, Carlos (2012): "Lavapiés. CSA/Os. Centros Sociales Autogestionados y/o Okupados", en Agustín Hernández Aja (coord.), $R e-h a b$, regeneración urbana integrada (Madrid 28-30 de octubre de 2012). Madrid: ETSAM.

VILLEGAS, Miguel (2012): "Sumario de Arquitectura Viva 145: Colectivos Españoles" [en línea], en arquitextonica, vol. octubre 2012. En: http://arquitextonica.net/2012/10/26/revistas-arquitectura-viva-145colectivos-espanoles/ [Consulta: diciembre de 2012]. 